\title{
CONVERGENCE OF THE RANDOM VORTEX METHOD IN TWO DIMENSIONS
}

\author{
DING-GWO LONG
}

\section{INTRODUCTION}

In this paper we prove an almost optimal rate of convergence of the random vortex method in two dimensions through a simple and unified approach. The framework of the proof can easily be adapted to treat the time discretizations and the convergence of the random vortex method in three dimensions $[12,13]$. It may also be applicable to analyze the accuracy of other numerical methods which involve random samplings.

The inviscid and the random vortex method with regularized velocity kernels were introduced by Chorin [5] to simulate inviscid and viscous incompressible fluid flows, respectively. The convergence of the inviscid vortex method was first proved by Hald [11], Beale and Majda [3, 4] for two and three dimensions. Later Cottet [6] and Anderson and Greengard [1] gave simpler proofs for the estimate of the consistency error.

The (time discretized) random vortex method in [5] is based on viscous splitting under which the Navier-Stokes equations are split into Euler's equations and the heat equation. Then Euler's equations are simulated by the inviscid vortex method and the heat equation is simulated by independent Gaussian random walks of the vortices. Thus the random vortex method generalizes the inviscid vortex method by adding a random term-Gaussian random walks. The convergence of the viscous splitting was proved by Beale and Majda [2].

Marchioror and Pulvirenti [15] considered the (continuous time) random vortex method with the Gaussian random walks replaced by independent Wiener processes (Brownian motions) and proved a "propagation of chaos" (law of large numbers) type of result on the convergence. Their result is not satisfactory from the point of view of numerical computations since there is no rate of convergence. One needs to look for a "large deviation" type of result.

Received by the editors July 12, 1987.

1980 Mathematics Subject Classification (1985 Revision). Primary 65M15, 76C05; Secondary $60 \mathrm{~F} 10$.

Partially supported by (1) IBM Graduate Fellowship, (2) NSF Grant DMS 84-0223, (3) ARO Grant DAAL 03-86-K-0003. 
Goodman [10] proved a rate of convergence of the order $N^{-1 / 4} \ln N$ where $N$ is the number of vortices used in the computation. However, his approach has the following limitations.

(1) The rate of convergence is not optimal. It should be roughly of the order $N^{-1 / 2}$ by the central limit theorem.

(2) His approach requires the smoothing parameter $\delta$ to be of the order $N^{-1 / 4} \ln ^{2} N \sim h^{1 / 2}|\ln h|^{2}$ or greater where $h \sim N^{-1 / 2}$ is the grid size.

(3) There is no dependence of the sampling error on the viscosity $\nu$ since the initial positions of the vortices are chosen randomly.

In this paper we prove a rate of convergence of the order $N^{-1 / 2} \ln N \sim h|\ln h|$ uniformly for $\nu \in\left[0, \nu_{0}\right]$ with arbitrary $\nu_{0}>0$ while $\delta$ only has to be of the order $h^{q}$ or greater for any $0<q<1$. The problem of detailed $\nu$-dependence as $\nu \rightarrow 0$ will be treated in [14].

The papers by Hald [11], Beale and Majda [3, 4] (abbreviated as B-M hereafter), and Anderson and Greengard [1] (abbreviated as A-G hereafter) have established the foundation of analyzing the vortex method in two and three dimensions. Since the random vortex method is a random perturbation of the inviscid vortex method, a satisfactory analysis of the former should be a generalized version of the latter. Therefore, it is important to study how to incorporate the additional probabilistic features appropriately. The approach of this paper is based on B-M and A-G with suitable modifications in order to treat the sampling errors and the random motions of the vortices. Therefore, the proof presented here is more natural other than being almost optimal.

$\S \S 2,3$, and 4 provide the necessary backgrounds for the development in the later sections. They include a brief review of the inviscid vortex method, the formulation of the random vortex method as a system of interacting diffusion processes, the statement of the main result (Theorem 2), and a few estimates which will be applied throughout the later sections.

$\S \S 5,6$, and 7 focus on the proof of the main result. The proof consists of two parts: the consistency and the stability estimates. The key idea in the consistency part is to separate the sampling error from two other types of errorthe moment error and the discretization error, and then estimate it through Bennett's inequality which is an estimate of large deviation type. The moment error and the discretization error are essentially the same as their counterparts in the inviscid case. Lemma 1 in $\S 4$, an estimate for the quadrature errors, is established for analyzing the discretization error. It is a generalization of the quadrature estimate in A-G. It turns out that Lemma 1 is also needed in estimating the variance of the sampling error and the variances associated with the stability estimate. The key idea in the stability part is to prove that the density of the vortices is bounded so that one can still apply Calderón-Zygmund inequality as B-M did in the inviscid case. 


\section{THE INVISCID VORTEX METHOD}

Euler's equations for homogeneous incompressible inviscid fluid flows in $\mathbf{R}^{2}$ are

$$
\begin{gathered}
\frac{\partial u}{\partial t}+(u \cdot \nabla) u=-\nabla p, \\
\nabla \cdot u=0
\end{gathered}
$$

where $u(x, t)=\left(u_{1}(x, t), u_{2}(x, t)\right)$ is the velocity field with $x=\left(x_{1}, x_{2}\right)$ in $\mathbf{R}^{2}$. By taking the curl of equation (1), we obtain

$$
\frac{\partial \omega}{\partial t}+\nabla \cdot(\omega u)=0
$$

where $\omega=\partial u_{2} / \partial x_{1}-\partial u_{1} / \partial x_{2}$ is the vorticity. Equation (3) is the continuity equation for the vorticity $\omega$. Therefore, the total vorticity contained in any piece of fluid is conserved during the motion. Moreover, by the incompressibility condition (2), equation (3) reduces to

$$
\frac{\partial \omega}{\partial t}+(u \cdot \nabla) \omega=0,
$$

and since the volume of any fluid element is conserved, $\omega$ is conserved on particle paths. Namely,

$$
\omega(x(t ; \alpha), t)=\omega(\alpha, 0)
$$

where $x(t ; \alpha)$ is the solution of the ODE

$$
\frac{d x}{d t}=u(x, t)
$$

with the initial data $x(0 ; \alpha)=\alpha$ and $\alpha=\left(\alpha_{1}, \alpha_{2}\right)$ is the Lagrangian coordinates. Notice that $x(t ; \alpha)$ are the characteristic curves of equations (3) and (4). It follows from the incompressibility condition (2) that the vorticity determines the velocity. Since $\nabla \cdot u=0$, there exists a stream function $\Psi$ such that

$$
u=\left(\frac{\partial \Psi}{\partial x_{2}},-\frac{\partial \Psi}{\partial x_{1}}\right)
$$

and

$$
\omega=-\nabla^{2} \Psi
$$

Therefore $\Psi=G * \omega$ and

$$
u(x, t)=(K * \omega)(x, t)=\int_{\mathbf{R}^{2}} K\left(x-x^{\prime}\right) \omega\left(x^{\prime}, t\right) d x^{\prime}
$$

where $G(x)=-(2 \pi)^{-1} \ln |x|$ and

$$
K(x)=\left(\frac{\partial G}{\partial x_{2}}(x),-\frac{\partial G}{\partial x_{1}}(x)\right)=\frac{1}{2 \pi|x|^{2}}\left(-x_{2}, x_{1}\right) .
$$


By combining (6), (7), (3), and (5), we obtain an equivalent formulation of Euler's equations by the integro-differential equation

$$
\frac{d x}{d t}(t ; \alpha)=\int_{\mathbf{R}^{2}} K\left(x(t ; \alpha)-x\left(t ; \alpha^{\prime}\right)\right) \omega\left(\alpha^{\prime}, 0\right) d \alpha^{\prime}
$$

with the initial data

$$
x(0 ; \alpha)=\alpha \in \mathbf{R}^{2} .
$$

The vortex method is a discrete approximation of the particle trajectory formulation (8) with the kernel $K$ replaced by a mollified one, i.e., we use a finite number of particles (vortices) to replace the integro-differential equation (8) by a finite system of ordinary differential equations:

$$
\frac{d \tilde{x}_{i}}{d t}(t)=\sum_{j h \in \Lambda^{h}} K_{\delta}\left(\tilde{x}_{i}(t)-\tilde{x}_{j}(t)\right) \omega_{j} h^{2}
$$

with the initial data

$$
\tilde{x}_{i}(0)=\alpha_{i}
$$

where

$$
\begin{gathered}
K_{\delta}=K * \psi_{\delta}, \quad \psi_{\delta}(x)=\delta^{-2} \psi\left(\delta^{-1} x\right), \delta>0, \\
\int_{\mathbf{R}^{2}} \psi(x) d x=1, \quad \omega_{i}=\omega\left(\alpha_{i}, 0\right),
\end{gathered}
$$

the initial vorticity $\omega(\cdot, 0)$ has compact support, and

$$
\Lambda^{h}=\left\{\alpha_{i}: \alpha_{i}=h \cdot i=h \cdot\left(i_{1}, i_{2}\right), i \in \mathbf{Z}^{2}\right\}
$$

is a lattice with spacing $h>0$. Thus the continuous and the discrete velocities computed from the vortex method are

$$
\tilde{u}^{h}(x, t)=\sum_{j} K_{\delta}\left(x-\tilde{x}_{j}(t)\right) \omega_{j} h^{2}, \quad \tilde{u}_{i}^{h}(t)=\sum_{j} K_{\delta}\left(\tilde{x}_{i}(t)-\tilde{x}_{j}(t)\right) \omega_{j} h^{2} .
$$

The choice of the smoothing function $\psi$ is closely related to the accuracy of the method. Following B-M and A-G we denote $\psi \in M^{L, m}$ if it satisfies the three conditions below.

(i) $\int_{\mathbf{R}^{2}} \psi(x) d x=1$.

(ii) $\int_{\mathbf{R}^{2}} x^{\beta} \psi(x) d x=0$, for all multi-indices $\beta$ with $1 \leq|\beta| \leq m-1$.

(iii) $\psi \in C^{L}\left(\mathbf{R}^{2}\right)$ and $\psi$ decreases rapidly at infinity.

The numerical analysis of the inviscid vortex method consists of two partsconsistency and stability. One introduces a reference velocity $u^{h}(x, t)$ obtained from a finite sum

$$
u^{h}(x, t)=\sum_{j} K_{\delta}\left(x-x_{j}(t)\right) \omega_{j} h^{2}
$$

where $x_{j}(t)$ is the exact particle path which is the solution of the ODE (6) with the initial data $x_{j}(0)=h \cdot j$. The discrete reference velocity is denoted by

$$
u_{i}^{h}(t)=u^{h}\left(x_{i}(t), t\right)=\sum_{j} K_{\delta}\left(x_{i}(t)-x_{j}(t)\right) \omega_{j} h^{2} .
$$


The difference between the exact velocity $u$ and the velocity $\tilde{u}$ from the vortex method is bounded by

$$
\begin{aligned}
\left|\tilde{u}^{h}(x, t)-u(x, t)\right| & \leq\left|\tilde{u}^{h}(x, t)-u^{h}(x, t)\right|+\left|u^{h}(x, t)-u(x, t)\right| \\
& =\text { stability + consistency. }
\end{aligned}
$$

A-G gave a simpler proof of the consistency estimate that

$$
\max _{0 \leq t \leq T} \max _{x \in \mathbf{R}^{2}}\left|u^{h}(x, t)-u(x, t)\right| \leq C\left[\delta^{m}+(h / \delta)^{L} \delta\right]
$$

with the smoothing function $\psi \in M^{L, m}$. For the stability estimate, B-M proved that if $\max _{h \cdot j \in \Lambda^{h}}\left|\tilde{x}_{i}(t)-x_{i}(t)\right| \leq \delta$ for all $t, 0 \leq t \leq T_{*} \leq T$, then

and

$$
\left\|\tilde{u}_{i}^{h}(t)-u_{i}^{h}(t)\right\|_{L_{h}^{p}} \leq C\left\|\tilde{x}_{i}(t)-x_{i}(t)\right\|_{L_{h}^{p}}
$$

$$
\left\|\tilde{u}^{h}(\cdot, t)-u^{h}(\cdot, t)\right\|_{L^{p}\left(B\left(R_{0}\right)\right)} \leq C\left\|\tilde{x}_{i}(t)-x_{i}(t)\right\|_{L_{h}^{p}}
$$

uniformly for $t \in\left[0, T_{*}\right]$ where the discrete $L^{p}$ norm $\left\|v_{i}\right\|_{L_{h}^{p}}=\left(\sum_{i}\left|v_{i}\right|^{p} h^{2}\right)^{1 / p}$ and $B\left(R_{0}\right)$ is a ball of radius $R_{0}$.

By combining the consistency and the stability estimates, the main result on the convergence is obtained. It is stated in the following theorem.

Theorem 1. Assume that the velocity field $u(x, t)$ is smooth enough, that the initial vorticity has bounded support and $\psi \in M^{L, M}$. Then for all sufficiently small $h$ and $\delta, \delta \geq h^{q}, q<1$, we have the following estimates.

(i) Convergence of particle paths

$$
\max _{0 \leq t \leq T}\left\|\tilde{x}_{i}(t)-x_{i}(t)\right\|_{L_{h}^{p}} \leq C\left[\delta^{m}+(h / \delta)^{L} \delta\right] .
$$

(ii) Convergence of discrete velocity

$$
\max _{0 \leq t \leq T}\left\|\tilde{u}_{i}^{h}(t)-u\left(x_{i}(t), t\right)\right\|_{L_{h}^{p}} \leq C\left[\delta^{m}+(h / \delta)^{L} \delta\right] .
$$

(iii) Convergence of continuous velocity

$$
\max _{0 \leq t \leq T}\left\|\tilde{u}^{h}(\cdot, t)-u(\cdot, t)\right\|_{L^{m}\left(B\left(R_{0}\right)\right)} \leq C\left[\delta^{m}+(h / \delta)^{L} \delta\right] .
$$

Here $R_{0}>0$ is an arbitrary finite radius. The constant $C$ depends only on $T, L, m, p, q, R_{0}$, the diameter of $\operatorname{supp} \omega_{0}$, and the bounds for a finite number of derivatives of the velocity field $u(x, t)$.

\section{THE RANDOM VORTEX METHOD}

We begin with the formulation of the random vortex method as a system of interacting diffusion processes. The Navier-Stokes equations for incompressible viscous fluid flows are

$$
\begin{gathered}
\frac{\partial u}{\partial t}+(u \cdot \nabla) u=-\nabla p+\nu \nabla^{2} u, \\
\nabla \cdot u=0,
\end{gathered}
$$


where $\nu$ is the kinematic viscosity. The vorticity-stream formulation is

$$
\begin{aligned}
& \frac{\partial \omega}{\partial t}+(u \cdot \nabla) \omega=\nu \nabla^{2} \omega, \\
& u(x, t)=(K * \omega)(x, t) .
\end{aligned}
$$

Since $\nabla \cdot u=0,(10)$ can be written as

$$
\frac{\partial \omega}{\partial t}+\nabla \cdot(u \omega)=\nu \nabla^{2} \omega .
$$

In the terminology of probability theory, equation (10) is called a backward equation and equation (11) is called a forward equation. That $\omega(x, t)$ is a solution of both a backward and a forward equation implies that the maximum principle

$$
\sup _{x \in \mathbf{R}^{2}} \omega(x, t) \leq \sup _{x \in \mathbf{R}^{2}} \omega(x, s), \quad \text { for } t \geq s,
$$

is satisfied and the total vorticity

$$
\mathscr{V}(t)=\int_{\mathbf{R}^{2}} \omega(x, t) d x
$$

is conserved, respectively.

We assume that the velocity field $u(x, t)$ is sufficiently smooth. The random vortex method is motivated from the following probabilistic interpretation of (11). The appropriate generalization of particle paths for equation (11) is the diffusion process $X(t ; \alpha), t \geq 0$, defined by the stochastic differential equation (abbreviated as SDE)

$$
d X(t ; \alpha)=u(X(t ; \alpha), t) d t+\sqrt{2 \nu} d W(t)
$$

with the initial data

$$
X(0 ; \alpha)=\alpha,
$$

where $W(t)$ is a standard Wiener process (Brownian motion) in $\mathbf{R}^{2}$. Since the diffusion coefficient $\sqrt{2 \nu}$ in equation (12) is a constant, (12) is equivalent to the integral equation

$$
X(t ; \alpha)=\alpha+\int_{0}^{t} u(X(s ; \alpha), s) d s+\sqrt{2 \nu} W(t) .
$$

It is not necessary to introduce Ito's stochastic integral here. Ito's integration is needed for variable diffusion coefficients. Since $W(t)$ has continuous sample paths with probability one, (13) can be solved sample path by sample path. For each continuous sample path $\zeta(t)$ of the process $W(t)$, the (deterministic) integral equation

$$
\xi(t ; \alpha)=\alpha+\int_{0}^{t} u(\xi(s ; \alpha), s) d s+\sqrt{2 \nu} \zeta(t)
$$

has a unique continuous solution. We will utilize this special property in our proof of the convergence of the random vortex method. The basic relation between the parabolic PDE (11) and the SDE (12) is that the fundamental solution 
(Green's function) $G(x, t ; \alpha, s)$ of the PDE is the transition probability density of the diffusion process $X$, i.e., $G(x, t ; \alpha, s)$ is the probability density that a particle reaches the position $x$ at time $t$ from position $\alpha$ and time $s<t$. See Friedman [9, Theorem 5.4 on p. 149].

Suppose a probability density $p_{0}(x)$ in $\mathbf{R}^{d}$ is well approximated weakly by $N$ particles with equal weights $N^{-1}$ at time $t=0$, i.e., for a small cube centered at $x$ and with side length $\Delta x$,

$$
p_{0}(x) \cdot(\Delta x)^{d} \approx \Delta N / N
$$

for any $x \in \mathbf{R}^{d}$ where $\Delta N$ is the number of particles in the cube. If we let the particles move according to the diffusion process (13), then it is intuitively clear that the ensemble of $N$ particles at time $t>0$ approximates the probability density

$$
p(x, t)=\int G(x, t ; \alpha, 0) p_{0}(\alpha) d \alpha
$$

provided that $N$ is large enough. On the other hand, $p(x, t)$ is the unique solution of the PDE (11) with the initial data $p_{0}(x)$. This suggests a numerical method to compute $p(x, t)$ or any continuous functional of $p(x, t)$, like $\left(K_{\delta} * p\right)(x, t)$ by simulating the diffusion process $X$. For the random vortex method in two dimensions, the particles may carry different weights with positive or negative signs.

From the above discussion, the particle tranjectory formulation should be derived from the SDE (12). We can write the velocity in the following way:

$$
\begin{aligned}
u(x, t) & =\int_{\mathbf{R}^{2}} K(x-y) \omega(y, t) d y \\
& =\int_{\mathbf{R}^{2}} K(x-y)\left[\int_{\mathbf{R}^{2}} G(y, t ; \alpha, 0) \omega(\alpha, 0) d \alpha\right] d y \\
& =\int_{\mathbf{R}^{2}}\left[\int_{\mathbf{R}^{2}} K(x-y) G(y, t ; \alpha, 0) d y\right] \omega(\alpha, 0) d \alpha \\
& =\int_{\mathbf{R}^{2}} E[K(x-X(t ; \alpha)] \omega(\alpha, 0) d \alpha
\end{aligned}
$$

where $E Y$ denotes the expectation of the random vector $Y$. It follows that (12) can be rewritten as

$$
\begin{aligned}
d X(t ; \alpha)= & \left\{\int_{\mathbf{R}^{2}} E^{\prime}\left[K\left(X(t ; \alpha)-X\left(t ; \alpha^{\prime}\right)\right)\right] \omega\left(\alpha^{\prime}, 0\right) d \alpha^{\prime}\right\} d t \\
& +\sqrt{2 \nu} d W(t)
\end{aligned}
$$

where $E^{\prime} K\left(X(t ; \alpha)-X\left(t ; \alpha^{\prime}\right)\right)$ is $E K\left(x-X\left(t ; \alpha^{\prime}\right)\right)$ evaluated at $x=X(t ; \alpha)$. Equation (14) resembles closely the particle trajectory formulation (8) in the inviscid case. This leads naturally to the random vortex method where we replace $\tilde{x}_{i}(t)$ and $x_{i}(t)$ in the inviscid vortex method by the corresponding 
diffusion processes $\tilde{X}_{i}(t)$ and $X_{i}(t)$, respectively. $\tilde{X}_{i}(t)$ is the solution of the system of SDE's

$$
d \widetilde{X}_{i}(t)=\left\{\sum_{j} K_{\delta}\left(\widetilde{X}_{i}(t)-\tilde{X}_{j}(t)\right) \omega_{j} h^{2}\right\} d t+\sqrt{2 \nu} d W_{i}(t)
$$

with the initial data

$$
\tilde{X}_{i}(0)=\alpha_{i}=h \cdot i
$$

and $X_{i}(t)$ is the solution of the system of SDE's

$$
d X_{i}(t)=u\left(X_{i}(t), t\right) d t+\sqrt{2 \nu} d W_{i}(t)
$$

with the same initial data $X_{i}(0)=\alpha_{i}$ where $W_{i}(t)$ with $h \cdot i \in \Lambda^{h}$ are independent standard Wiener processes in $\mathbf{R}^{2}$. Our main result is the following theorem.

Theorem 2. Under the same assumption as in Theorem 1, we have the following estimates.

(i) Convergence of particle paths

$$
\max _{0 \leq t \leq T}\left\|\widetilde{X}_{i}(t)-X_{i}(t)\right\|_{L_{h}^{p}} \leq C\left[\delta^{m}+(h / \delta)^{L} \delta+h|\ln h|\right] .
$$

(ii) Convergence of discrete velocity

$$
\max _{0 \leq t \leq T}\left\|\tilde{u}_{i}^{h}(t)-u\left(X_{i}(t), t\right)\right\|_{L_{h}^{p}} \leq C\left[\delta^{m}+(h / \delta)^{L} \delta+h|\ln h|\right] .
$$

(iii) Convergence of continuous velocity

$$
\max _{0 \leq t \leq T}\left\|\tilde{u}^{h}(\cdot, t)-u(\cdot, t)\right\|_{L^{p}\left(B\left(R_{0}\right)\right)} \leq C\left[\delta^{m}+(h / \delta)^{L} \delta+h|\ln h|\right]
$$

except for an event of probability less than $h^{C^{\prime} C}$ provided that $C \geq C^{\prime \prime}$ where the constants $C^{\prime}, C^{\prime \prime}>0$ depend only on the same parameters as described in Theorem 1 .

Notice that one can choose a larger constant $C$ so that the probability $h^{C^{\prime} C}$ approaches zero faster. There is a trade off between the accuracy and the probability.

Notation. We will use the short hand $a \preceq b$ in the rest of this paper to denote $a \leq b$ except for an event of probability approaching zero faster than any polynomial rate by choosing the constant $C$ sufficiently large.

\section{Preliminary estimates}

Since the initial positions of the vortices are chosen on the lattice points instead of being chosen randomly, the following lemma for the quadrature error is essential to our analysis. It generalizes the corresponding inviscid estimate in A-G to the viscous case $\nu>0$. 
Lemma 1. Let $X(t ; \alpha)$ be the solution of the SDE

$$
d X(t ; \alpha)=u(X(t ; \alpha), t) d t+\sqrt{2 \nu} d W(t)
$$

in $\mathbf{R}^{d}$ with the initial data $X(0 ; \alpha)=\alpha \in \mathbf{R}^{d}$ where $u \in C^{L}\left(\mathbf{R}^{d} \times[0, T]\right)$, $L \geq d+1$ and all the spatial derivatives of $u(\cdot, t)$ up to the order $L$ are uniformly bounded. Define $\Gamma(\alpha, t)=\{E f(X(t ; \alpha))\} \cdot g(\alpha)$ with $\mathbf{R}^{d^{\prime}}$-valued functions $f \in C_{b}^{L}\left(\mathbf{R}^{d}\right), g \in C_{0}^{L}\left(\mathbf{R}^{d}\right), L \geq d+1$. Consequently the support of $\Gamma$ is contained in a bounded domain $\Omega \supset \operatorname{supp} g$. Then we have the following estimate for the quadrature error

$$
\begin{aligned}
\max _{0 \leq t \leq T} \mid & \sum_{i \in \mathbf{Z}^{d}} \Gamma(h \cdot i, t) h^{d}-\int_{\mathbf{R}^{d}} \Gamma(\alpha, t) d \alpha \mid \\
\leq & C h^{L} \max _{0 \leq|\beta| \leq L}\left\|\partial^{\beta} g\right\|_{L^{\infty}} \\
& \times\left\{\sum_{0 \leq|\beta| \leq L} \int_{|x| \leq R}\left|\partial^{\beta} f(x)\right| d x+\sum_{0 \leq|\beta| \leq L} \sup _{|x|>R}\left|\partial^{\beta} f(x)\right|\right\}
\end{aligned}
$$

where $R>0$ is arbitrary and $C$ depends only on $d, d^{\prime}, T, L$, the diameter of $\Omega$, and $\max _{1 \leq|\gamma| \leq L}\left\|\partial^{\gamma} u\right\|_{L^{\infty}\left(\mathbf{R}^{d} \times[0, T]\right)}$.

The proof is very similar to the inviscid case. We follow the approach in Cottet [6] and A-G which requires the following lemma.

Lemma 2. Let $f \in C_{0}^{L}\left(\mathbf{R}^{d}\right)$. Then for $L \geq d+1$ we have

$$
\left|\sum_{i \in \mathbf{Z}^{d}} f(h \cdot i) h^{d}-\int_{\mathbf{R}^{d}} f(x) d x\right| \leq C h^{L} \cdot \max \left(\left\|\partial_{1}^{L} f\right\|_{L^{1}, \ldots .}\left\|\partial_{d}^{L} f\right\|_{L^{1}}\right),
$$

where the constant $C$ depends only on $d$.

See p. 424 of A-G for a proof.

Proof of Lemma 1 . We prove the case that $d=2$ and $f, g$ are real valued. The proof for the general cases is essentially the same. In order to apply Lemma 2, we need to estimate $\left\|\partial_{1}^{L} \Gamma\right\|_{L^{1}}$ and $\left\|\partial_{2}^{L} \Gamma\right\|_{L^{1}}$. By direct differentiation we know that $\partial_{1}^{L} \Gamma$ and $\partial_{2}^{L} \Gamma$ are sums of finite terms of the form

$$
E\left[\left(\partial^{\beta} f\right)(X(t ; \alpha)) \cdot \prod_{1 \leq|\gamma| \leq L}\left(\partial^{\gamma} X(t ; \alpha)\right)^{\kappa(\gamma)}\right] \partial^{\mu}(g(\alpha)
$$

where $\beta, \gamma, \kappa, \mu$ are multiple indices with $0 \leq|\beta|,|\gamma|,|\kappa|,|\mu| \leq L$. We need a bound for $\partial^{\gamma} X(t ; \alpha)$. By differentiating

$$
X(t ; \alpha)=\alpha+\int_{0}^{t} u(X(s ; \alpha), s) d s+\sqrt{2 \nu} W(t),
$$


we obtain integral equations

$$
\begin{aligned}
\frac{\partial}{\partial \alpha_{1}} X(t ; \alpha) & =\left(\begin{array}{l}
1 \\
0
\end{array}\right)+\int_{0}^{t}[\nabla u(X(s ; \alpha), s)] \cdot\left[\frac{\partial}{\partial \alpha_{1}} X(s ; \alpha)\right] d s \\
\frac{\partial}{\partial \alpha_{2}} X(t ; \alpha) & =\left(\begin{array}{l}
0 \\
1
\end{array}\right)+\int_{0}^{t}[\nabla u(X(s ; \alpha), s)] \cdot\left[\frac{\partial}{\partial \alpha_{2}} X(s ; \alpha)\right] d s
\end{aligned}
$$

for $\left(\partial / \partial \alpha_{1}\right) X(t ; \alpha)$ and $\left(\partial / \partial \alpha_{2}\right) X(t ; \alpha)$. It follows that

$$
\begin{aligned}
\left|\frac{\partial}{\partial \alpha_{1}} X(t ; \alpha)\right| & \leq 1+\int_{0}^{t}\|\nabla u\|_{L^{\infty}} \cdot\left|\frac{\partial}{\partial \alpha_{1}} X(s ; \alpha)\right| d s, \\
\left|\frac{\partial}{\partial \alpha_{2}} X(t ; \alpha)\right| & \leq 1+\int_{0}^{t}\|\nabla u\|_{L^{\infty}} \cdot\left|\frac{\partial}{\partial \alpha_{2}} X(s ; \alpha)\right| d s .
\end{aligned}
$$

Therefore

$$
\left|\frac{\partial}{\partial \alpha_{1}} X(t ; \alpha)\right|,\left|\frac{\partial}{\partial \alpha_{2}} X(t ; \alpha)\right| \leq \exp \left(\|\nabla u\|_{L^{\infty}\left(\mathbf{R}^{2} \times[0, T]\right)} T\right)
$$

by Gronwall's inequality.

The integral equation for higher order derivatives $\partial^{\gamma} X(t ; \alpha)$ are

$$
\partial^{\gamma} X(t ; \alpha)=\int_{0}^{t} Y(s) d s+\int_{0}^{t}[\nabla u(X(s ; \alpha), s)] \cdot \partial^{\gamma} X(s ; \alpha) d s
$$

where $Y(s)$ is some function of $\left(\partial^{\lambda} u\right)(X(s ; \alpha))$ and $\partial^{\rho} X(s ; \alpha)$ with $1 \leq|\lambda| \leq$ $|\gamma|$ and $1 \leq|\rho| \leq|\gamma-1|$. By Gronwall's inequality and induction on $\gamma$, we conclude that

$$
\left|\partial^{\gamma} X(t ; \alpha)\right| \leq C, \quad 1 \leq|\gamma| \leq L
$$

where $C$ depends only on $\max _{1 \leq|\gamma| \leq L}\left\|\partial^{\gamma} u\right\|_{L^{\infty}\left(\mathbf{R}^{2} \times[0, T]\right)}, L$, and $T$.

It follows from (18) and (19) that

$$
\begin{aligned}
\left\|\partial_{1}^{L} \Gamma\right\|_{L^{1}} & =\int_{\mathbf{R}^{2}}\left|\frac{\partial^{L}}{\partial \alpha_{1}^{L}} \Gamma(\alpha)\right| d \alpha=\int_{\Omega}\left|\frac{\partial^{L}}{\partial \alpha_{1}^{L}} \Gamma(\alpha)\right| d \alpha \\
& \leq C \max _{0 \leq|\beta| \leq L}\left\|\partial^{\beta} g\right\|_{L^{\infty}} \sum_{0 \leq|\beta| \leq L} \int_{\Omega}\left|E\left(\partial^{\beta} f\right)(X(t ; \alpha))\right| d \alpha \\
& \leq C \max _{0 \leq|\beta| \leq L}\left\|\partial^{\beta} g\right\|_{L^{\infty}} \sum_{0 \leq|\beta| \leq L} \int_{\Omega} E\left|\left(\partial^{\beta} f\right)(X(t ; \alpha))\right| d \alpha .
\end{aligned}
$$

Similarly, $\left\|\partial_{2}^{L} \Gamma\right\|_{L^{1}}$ is bounded by (20). One can write

$$
\begin{aligned}
\int_{\Omega} E\left|\left(\partial^{\beta} f\right)(X(t ; \alpha))\right| d \alpha & =\int_{\Omega} \int_{\mathbf{R}^{2}}\left|\partial^{\beta} f(x)\right| G(x, t ; \alpha, 0) d x d \alpha \\
& =\int_{\mathbf{R}^{2}}\left|\partial^{\beta} f(x)\right|\left[\int_{\Omega} G(x, t ; \alpha, 0) d \alpha\right] d x
\end{aligned}
$$


where $G$ is the fundamental solution of the vorticity equation (11). The function $v(x, t)=\int_{\Omega} G(x, t ; \alpha, 0) d \alpha$ is the solution of the vorticity equation (11) with the initial data $v(\cdot, 0)=\mathscr{X}_{\Omega}$ where

$$
\mathscr{X}_{\Omega}= \begin{cases}1 & \text { if } x \in \Omega \\ 0 & \text { if } x \notin \Omega\end{cases}
$$

is the characteristic function of $\Omega$. Since $v(x, t)$ satisfies the backward equation (10) and the forward equation (11), we have

$$
0<v(x, t)<1 \text { and } \int_{\mathbf{R}^{2}} v(x, t) d x=\operatorname{Area}(\Omega), \forall t>0
$$

by the maximum principle and the conservation of vorticity, respectively. Therefore (21) can be estimated by

$$
\begin{aligned}
\int_{\mathbf{R}^{2}} & \left|\partial^{\beta} f(x)\right|\left[\int_{\Omega} G(x, t ; \alpha, 0) d \alpha\right] d x \\
& =\int_{\mathbf{R}^{2}}\left|\partial^{\beta} f(x)\right| \cdot v(x, t) d x \\
& \leq \int_{|x| \leq R}\left|\partial^{\beta} f(x)\right| d x+\operatorname{Area}(\Omega) \cdot \sup _{|x|>R}\left|\partial^{\beta} f(x)\right| .
\end{aligned}
$$

The lemma is proved by combining (20), (21), (22), and Lemma 2.

Since the Navier-Stokes equations are nonlinear, we need a uniform bound on the velocities $u$ with respect to the viscosity $\nu$ ranging in a compact set (say, $0 \leq \nu \leq 1$ ) so that in later sections we can apply Lemma 1 to obtain estimates uniform in that compact set. The next lemma from Beale and Majda [2] provides the bound.

Lemma 3. Let $u^{\nu}$ denote a family of solutions of the Navier-Stokes equations with the same initial data $u_{0} \in H^{s}\left(\mathbf{R}^{2}\right), \nabla \times u_{0} \in L^{1}\left(\mathbf{R}^{2}\right), s \geq 4$, where $\nu$ is the viscosity. In particular, $u^{0}$ is the solution of Euler's equations. If $T$ is an arbitrarily large time interval, then

$$
\max _{\substack{0 \leq t \leq T \\ 0 \leq \nu \leq 1}}\left\|u^{\nu}(\cdot, t)\right\|_{H^{s}} \leq C
$$

where $C$ depends only on $T,\left\|u_{0}\right\|_{H^{s}}$, and $\left\|\nabla \times u_{0}\right\|_{L^{1}}$.

By Lemma 3 and Sobolev's lemma, $\max _{1 \leq|\beta| \leq L}\left\|\partial^{\beta} u\right\|_{L^{\infty}\left(\mathbf{R}^{2} \times[0, T]\right)}$ is uniformly bounded with respect to the viscosity $\nu$ if the initial data is smooth enough.

We also need the following estimates about $K_{\delta}$ and its derivatives.

Lemma 4. (i) $\left|\partial^{\beta} K_{\delta}(x)\right| \leq C_{\beta} \delta^{-1-|\beta|}, \forall x \in \mathbf{R}^{2}$.

(ii) $\left|\partial^{\beta} K_{\delta}(x)\right| \leq C_{\beta}|x|^{-1-|\beta|}, \forall|x| \geq \delta$.

See (5.7) and (5.8) on p. 47 of B-M. 
Lemma 5. (i) $\int_{|x| \leq R}\left|K_{\delta}(x)\right| d x \leq C R, \forall \delta<1$.

(ii) $\int_{|x| \leq R}\left|\partial^{\beta} K_{\delta}(x)\right| d x \leq C \ln (R / \delta)$, for $|\beta|=1, \delta<1$.

(iii) $\int_{|x| \leq R}\left|\partial^{\beta} K_{\delta}(x)\right| d x \leq C_{R} \delta^{1-|\beta|}$, for $|\beta|>1, \delta<1$.

See (5.9) on p. 47 of B-M and Lemma 2.3 on p. 425 of A-G.

The next lemma is useful in estimating the sampling error. It is also needed in proving the stability lemma.

Lemma 6 (Bennett's inequality). Let $Y_{i}$ be independent bounded random variables (not necessarily identically distributed) with mean zero, variances $\sigma_{i}^{2}$ and $\left|Y_{i}\right| \leq M$. Let $S=\sum_{i} Y_{i}, V \geq \sum_{i} \sigma_{i}^{2}$. Then for all $\eta>0$,

$$
P\{|S| \geq \eta\} \leq 2 \exp \left[-\frac{1}{2} \eta^{2} V^{-1} B\left(M \eta V^{-1}\right)\right]
$$

where $B(\lambda)=2 \lambda^{-2}[(1+\lambda) \ln (1+\lambda)-\lambda], \lambda>0, \lim _{\lambda \rightarrow 0^{+}} B(\lambda)=1$, and $B(\lambda) \sim 2 \lambda^{-1} \ln \lambda$ as $\lambda \rightarrow \infty$.

See Pollard [16, Appendix B] for a proof. For random vectors $Y_{i}$ in $\mathbf{R}^{2}$, we have

$$
P\{|S| \geq \eta\} \leq 4 \exp \left[-\frac{1}{4} \eta^{2} V^{-1} B\left(M \eta V^{-1}\right)\right]
$$

by applying (23) to the two components.

\section{CONSISTENCY ERROR FOR A FIXED TIME}

The consistency error can be decomposed into three components as follows.

$$
\begin{aligned}
\left|u^{h}(x, t)-u(x, t)\right|= & \left|\sum_{i} K_{\delta}\left(x-X_{i}(t)\right) \omega_{i} h^{2}-u(x, t)\right| \\
\leq & \left|\sum_{i} K_{\delta}\left(x-X_{i}(t)\right) \omega_{i} h^{2}-\sum_{i} E K_{\delta}\left(x-X_{i}(t)\right) \omega_{i} h^{2}\right| \\
& +\left|\sum_{i} E K_{\delta}\left(x-X_{i}(t)\right) \omega_{i} h^{2}-\int_{\mathbf{R}^{2}} K_{\delta}(x-y) \omega(y, t) d y\right| \\
& +\left|\int_{\mathbf{R}^{2}} K_{\delta}(x-y) \omega(y, t) d y-\int_{\mathbf{R}^{2}} K(x-y) \omega(y, t) d y\right| \\
= & \text { sampling error }+ \text { discretization error + moment error. }
\end{aligned}
$$

The moment error is exactly the same as in the inviscid case and it is proved in $B-M$ and $A-G$ that

$$
\left|\int_{\mathbf{R}^{2}} K_{\delta}(x-y) \omega(y, t) d y-\int_{\mathbf{R}^{2}} K(x-y) \omega(y, t) d y\right| \leq C \delta^{m} .
$$

For the discretization error we notice that $\int K_{\delta}(x-y) \omega(y, t) d y$ is a disguised version of $\int E\left[K_{\delta}(x-X(t ; \alpha))\right] \omega(\alpha, 0) d \alpha$. By applying Lemma 1 with 


$$
\begin{aligned}
& f(y)=K_{\delta}(x-y), g(\alpha)=\omega(\alpha, 0), \text { we obtain } \\
& \left|\sum_{i} E K_{\delta}\left(x-X_{i}(t)\right) \omega_{i} h^{2}-\int_{\mathbf{R}^{2}} E\left[K_{\delta}(x-X(t ; \alpha))\right] \omega(\alpha, 0) d \alpha\right| \\
& \quad \leq C h^{L}\left\{\sum_{0 \leq|\gamma| \leq L} \int_{|x-y| \leq R}\left|\partial^{\gamma} K_{\delta}(x-y)\right| d y+\sum_{0 \leq|\gamma| \leq L} \sup _{|x-y|>R}\left|\partial^{\gamma} K_{\delta}(x-y)\right|\right\} .
\end{aligned}
$$

We choose $R=\max (1, \operatorname{diameter}(\Omega))$. By Lemmas 4 and 5 ,

$$
\left|\sum_{i} E K_{\delta}\left(x-X_{i}(t)\right) \omega_{i} h^{2}-\int_{\mathbf{R}^{2}} K_{\delta}(x-y) \omega(y, t) d y\right| \leq C(h / \delta)^{L} \delta
$$

where the constant $C$ depends only on $L$, the $L^{\infty}$ bounds of the velocity field $u(x, t)$ and its spatial derivatives up to the order $L+1$, and the diameter of $\Omega$.

The main step in this section is to estimate the sampling error by applying Bennett's inequality. Let

$$
Y_{i}=\omega_{i} h^{2}\left[K_{\delta}\left(x-X_{i}(t)\right)-E K_{\delta}\left(x-X_{i}(t)\right)\right]
$$

We have $E Y_{i}=0,\left|Y_{i}\right| \leq C h^{2} \delta^{-1} \stackrel{\text { def }}{=} M$, and

$$
\begin{aligned}
\sum_{i} \operatorname{Var} Y_{i} & =h^{2} \sum_{i}\left\{E\left|K_{\delta}\left(x-X_{i}(t)\right)\right|^{2}-\left|E K_{\delta}\left(x-X_{i}(t)\right)\right|^{2}\right\} \omega_{i}^{2} h^{2} \\
& \leq h^{2} \sum_{i}\left\{E\left|K_{\delta}\left(x-X_{i}(t)\right)\right|^{2}\right\} \omega_{i}^{2} h^{2} .
\end{aligned}
$$

Here we simply drop the term $\left|E K_{\delta}\left(x-X_{i}(t)\right)\right|^{2}$ and consequently there is no dependence of the sampling errors on the viscosity $\nu$. The term will be taken into account in [14] to extract $\nu$-dependence. We apply Lemma 1 again with $f(y)=\left|K_{\delta}(x-y)\right|^{2}, g(\alpha)=\omega^{2}(\alpha, 0)$ to approximate $\sum_{i} E \mid K_{\delta}(x-$ $\left.X_{i}(t)\right)\left.\right|^{2} \omega_{i}^{2} h^{2}$ by the integral

$$
\int_{\Omega} E\left|K_{\delta}(x-X(t ; \alpha))\right|^{2} \omega^{2}(\alpha, 0) d \alpha
$$

with an error of $C(h / \delta)^{L}$ which follows from Lemmas 4 and 5 since $|K(x)|^{2}=$ constant $\cdot|\nabla K(x)|$. Notice that

$$
\begin{aligned}
\int_{\Omega} E \mid K_{\delta} & \left.(x-X(t ; \alpha))\right|^{2} \omega^{2}(\alpha, 0) d \alpha \\
= & \int_{\Omega} \int_{\mathbf{R}^{2}}\left|K_{\delta}(x-y)\right|^{2} G(y, t ; \alpha, 0) \omega^{2}(\alpha, 0) d y d \alpha \\
= & \int_{\mathbf{R}^{2}}\left|K_{\delta}(x-y)\right|^{2}\left\{\int_{\Omega} G(y, t ; \alpha, 0) \omega^{2}(\alpha, 0) d \alpha\right\} d y
\end{aligned}
$$

where $G$ is the Green's function. Since $\int_{\Omega} G(y, t ; \alpha, 0) \omega^{2}(\alpha, 0) d \alpha$ is a solution of both the forward and the backward equations, its $L^{\infty}$ and $L^{1}$ norm 
are bounded by those of the initial data respectively. Therefore, by choosing $R=\max (1$, diameter $(\Omega)),(24)$ is bounded by

$$
\begin{aligned}
& C_{1}\left\{\int_{|x-y| \leq R}\left|K_{\delta}(x-y)\right|^{2} d y+\sup _{|x-y|>R}\left|K_{\delta}(x-y)\right|^{2}\right\} \\
& \quad=C_{1}\left\{\int_{0 \leq|x| \leq \delta}\left|K_{\delta}(x)\right|^{2} d x+\int_{\delta \leq|x| \leq R}\left|K_{\delta}(x)\right|^{2} d x+C_{2}\right\} \\
& \quad \leq C_{3}\left\{\delta^{-2} \cdot \delta^{2}+\ln \frac{R}{\delta}+C_{2}\right\} \\
& \quad \leq C_{4}|\ln \delta|
\end{aligned}
$$

and we have

$$
\sum_{i} \operatorname{Var} Y_{i} \leq C h^{2}|\ln \delta| \stackrel{\text { def }}{=} V
$$

where the constant $C$ depends only on $L$, the $L^{\infty}$ bounds of the velocity field $u(x, t)$ and its spatial derivatives up to the order $L+1$, and the diameter of $\Omega$. It follows from Bennett's inequality that

$$
\begin{aligned}
P\{\mid & \left.\sum_{i} Y_{i}|\geq C h| \ln h \mid\right\} \\
& \leq 4 \exp \left\{-\frac{1}{4} C^{2}(h|\ln h|)^{2} V^{-1} B\left[M(C h|\ln h|) V^{-1}\right]\right\} \\
& \leq \exp \left\{-C_{1} C^{2}|\ln h|^{2}|\ln \delta|^{-1} \cdot B\left[C_{2} C h \delta^{-1}|\ln h||\ln \delta|^{-1}\right]\right\} \\
& \leq \exp \left\{-C_{3} C|\ln h|^{2}|\ln \delta|^{-1}\right\} \\
& \leq \exp \left\{-C_{3} C|\ln h|\right\} \quad(\text { by } h \leq \delta) \\
& =h^{C_{3} C} .
\end{aligned}
$$

In summary, we have proved an estimate for the consistency error at fixed $x$ and $t$ :

$$
\left|u^{h}(x, t)-u(x, t)\right| \leq C\left[\delta^{m}+(h / \delta)^{L} \delta+h|\ln h|\right]
$$

except for an event of probability less than $h^{C_{3} C}$ with $C_{3}>0$. For the lattice points $z_{k}=h \cdot k$ in any ball $B\left(R_{0}\right)$, it follows from the pointwise estimate (25) that

$$
\max _{k}\left|u^{h}\left(z_{k}, t\right)-u\left(z_{k}, t\right)\right| \leq C\left[\delta^{m}+(h / \delta)^{L} \delta+h|\ln h|\right]
$$

except for an event of probability less than $C_{4} h^{-2} \cdot h^{C_{3} C} \leq h^{C_{5} C}$ with some constant $C_{5}>0$ provided that $C$ is large enough. Let $X_{i}^{\prime}$ be an independent copy of $X_{i}$. It follows from $(25)$ and $K_{\delta}(0)=0$ that $\max _{i}\left|u_{i}^{h}(t)+K_{\delta}\left(X_{i}(t)-X_{i}^{\prime}(t)\right) \omega_{i} h^{2}-u\left(X_{i}(t), t\right)\right| \preceq C\left[\delta^{m}+(h / \delta)^{L} \delta+h|\ln h|\right]$.

Since $\left|K_{\delta}(x)\right| \leq C \delta^{-1}$,

$$
\max _{i}\left|u_{i}^{h}(t)-u\left(X_{i}(t), t\right)\right| \preceq C\left[\delta^{m}+(h / \delta)^{L} \delta+h|\ln h|\right] .
$$


(26) and (27) are the consistency estimates for the discrete velocities at any fixed time $t$.

\section{Stability estimate for all time}

The main stability estimate is stated in the following lemma.

Lemma 7 (Stability Lemma). Assume

$$
\max _{0 \leq t \leq T_{*}} \max _{h \cdot i \in \Lambda^{h}}\left|\tilde{X}_{i}(t)-X_{i}(t)\right| \preceq C_{0} \delta
$$

for some $T_{*} \leq T$, then

$$
\left\|\tilde{u}_{i}^{h}(t)-u_{i}^{h}(t)\right\|_{L_{h}^{p}} \preceq C\left\|\widetilde{X}_{i}(t)-X_{i}(t)\right\|_{L_{h}^{p}}
$$

uniformly for $t \in\left[0, T_{*}\right]$ where the constant $C$ depends only on $T, L, p, q$, the diameter of supp $\omega_{0}$, and the bounds for a finite number of derivatives of the velocity field $u . C$ is independent of $T_{*}$.

One can write

$$
\begin{aligned}
\tilde{u}_{i}^{h}-u_{i}^{h}= & \sum_{j}\left[K_{\delta}\left(X_{i}-\tilde{X}_{j}\right)-K_{\delta}\left(X_{i}-X_{j}\right)\right] \omega_{j} h^{2} \\
& +\sum_{j}\left[K_{\delta}\left(\widetilde{X}_{i}-\widetilde{X}_{j}\right)-K_{\delta}\left(X_{i}-\tilde{X}_{j}\right) \omega_{j} h^{2}\right. \\
= & v_{i}^{(1)}+v_{i}^{(2)} .
\end{aligned}
$$

In B-M the estimate for $v_{i}^{(1)}$ is accomplished by considering a partition of the support of $\omega(\cdot, t)$ into cells $B_{i}=\Phi^{t}\left(Q_{i}\right)$ where $\Phi^{t}$ is the flow map determined by the velocity field $u$ and $Q_{i}$ is the square centered at $h \cdot i$ with its sides of length $h$ and parallel to the axes. $v_{i}^{(1)}$ is then approximated by the integral

$$
v^{(1)}(x)=\int \mathscr{K}\left(x, x^{\prime}\right) f\left(x^{\prime}\right) d x^{\prime}
$$

where $v^{(1)}(x)=v_{i}^{(1)}$ for $x \in B_{i}, f\left(x^{\prime}\right)=e_{j} \omega_{j}, e_{j}=\tilde{x}_{j}-x_{j}$ for $x^{\prime} \in B_{j}$, and $\mathscr{K}\left(x, x^{\prime}\right)=\nabla K_{\delta}\left(x_{i}-x_{j}+y_{i j}\right)$ for $x \in B_{i}, x^{\prime} \in B_{j}$. Since $\Phi^{t}$ is area preserving, it follows that $\operatorname{Area}\left(B_{i}\right)=h^{2},\left\|v^{(1)}\right\|_{L^{p}}=\left\|v_{i}^{(1)}\right\|_{L_{h}^{p}}$, and $\|f\|=$ $\left\|e_{j} \omega_{j}\right\|_{L_{h}^{p}}$. This enables one to apply Calderón-Zygmund inequality to estimate $v_{i}^{(1)}$ after approximating $\mathscr{K}\left(x, x^{\prime}\right)$ by $\nabla K_{\delta}\left(x-x^{\prime}\right)$.

For the random vortex method, the approach is not readily applicable without modification since the positions of the vortices $\widetilde{X}_{i}(t), X_{i}(t)$ are random. Actually, it is not necessary to use the flow map to define a partition of $\mathbf{R}^{2}$. All we need is a partition satisfying

(1) the sizes of the cells being of the order $\delta$ or less,

(2) the densities of the vortices in the cells being uniformly bounded with high probability, 
so that one can still apply the Calderón-Zygmund inequality to estimate $v_{i}^{(1)}$. An obvious choice of the partition is the squares $Q_{k}$ centered at the lattice points $\delta \cdot k$ and with the side length $\delta$ where $k \in \mathbf{Z}^{2}$. The following lemma is about the uniform boundedness of the density of the vortices in balls. It is equivalent to the uniform boundedness in squares.

Lemma 8. Let $\mathscr{N}(x, r, t)=\sharp\left\{X_{i}(t):\left|X_{i}(t)-x\right| \leq r\right\}$, with $r>0,0 \leq t \leq T$, be the number of vortices in the ball $B(x, r)$. If $r \geq h|\ln h|$, then

$$
h^{2} \cdot \mathscr{N}(x, r, t) \preceq C r^{2} .
$$

Proof. Let

$$
H(y)= \begin{cases}1 & \text { if } y \in B(x, r), \\ 0 & \text { if } y \notin B(x, r)\end{cases}
$$

be the characteristic function of the ball $B(x, r)$. We can write

$$
\begin{aligned}
h^{2} \cdot \mathcal{N}(x, r, t) & =h^{2} \sum_{i} H\left(X_{i}(t)\right) \\
& =h^{2} \sum_{i} E H\left(X_{i}(t)\right)+h^{2} \sum_{i}\left\{H\left(X_{i}(t)\right)-E H\left(X_{i}(t)\right)\right\} \\
& =(I)+(I I)
\end{aligned}
$$

where $(I)$ is $h^{2}$ times the expected number of vortices inside $B(x, r)$ and $(I I)$ is the fluctuation from the expectation. In order to apply Lemma 1, we need to introduce a smooth function $\widetilde{H} \geq H$. Let $\phi \in C_{0}^{\infty}\left(\mathbf{R}^{2}\right)$ be defined by

$$
\phi(x)= \begin{cases}\exp \left(-x^{2} /\left(1-x^{2}\right)\right) & \text { if }|x|<1, \\ 0 & \text { if }|x| \geq 1 .\end{cases}
$$

Define the function $\widetilde{H}$ by

$$
\widetilde{H}(y)= \begin{cases}H(y)=1 & \text { if } y \in B(x, r), \\ \phi(|y-x| / r-1) & \text { if } r \leq|y-x| \leq 2 r, \\ 0 & \text { therwise. }\end{cases}
$$

It is obvious that $\widetilde{H} \in C_{0}^{\infty}\left(\mathbf{R}^{2}\right)$, and its partial derivatives of order $L$ are bounded by $C r^{-L}$ where $C$ depends only on $L$. Since $\widetilde{H} \geq H$, we can bound $(I)$ by $h^{2} \sum_{i} E \widetilde{H}\left(X_{i}(t)\right)$.

By applying Lemma 1 with $f(y)=\widetilde{H}(y), g(\alpha)=1$, we can approximate $h^{2} \sum_{i} E \widetilde{H}\left(X_{i}(t)\right)$ by the integral $\int_{\Omega} E \widetilde{H}(X(t ; \alpha)) d \alpha$ within an error

$$
C_{1} h^{L} \cdot 4 \pi r^{2} \sum_{l=0}^{L} r^{-l} \leq C_{2} r^{2} h^{L}\left\{L \cdot[\min (1, r)]^{-L}\right\} \leq C r^{2}
$$

provided that $h \leq r$. Moreover

$$
\begin{aligned}
\int_{\Omega} E \widetilde{H}(X(t ; \alpha)) d \alpha & \leq \int_{\Omega} \int_{B(x, 2 r)} G(y, t ; \alpha, 0) d y d \alpha \\
& \leq \int_{B(x, 2 r)} d y=4 \pi r^{2}
\end{aligned}
$$


Thus we arrive at the conclusion that $(I) \mid \leq C r^{2}$ if $r \geq h$.

We use Bennett's inequality to estimate $(I I)$. Let

$$
Y_{i}=h^{2}\left[H\left(X_{i}(t)\right)-E H\left(X_{i}(t)\right)\right] .
$$

We have $E Y_{i}=0,\left|Y_{i}\right| \leq h^{2}$, and

$$
\sum_{i} \operatorname{Var} Y_{i} \leq h^{4} \sum_{i} E\left[H\left(X_{i}(t)\right)\right]^{2} \leq h^{4} \sum_{i} E\left[\widetilde{H}\left(X_{i}(t)\right)\right]^{2} .
$$

We apply Lemma 1 with $f(y)=E\left[\widetilde{H}\left(X_{i}(t)\right)\right]^{2}, g(\alpha)=1$, to approximate $h^{2} \sum_{i} E\left[\widetilde{H}\left(X_{i}(t)\right)\right]^{2}$ by the integral $\int_{\Omega} E[\tilde{H}(X(t ; \alpha))]^{2} d \alpha$ within an error

$$
C_{1} h^{L} \cdot 4 \pi r^{2} \sum_{l=0}^{L} r^{-l} \leq C_{2} r^{2} h^{L}\left\{L \cdot[\min (1, r)]^{-L}\right\} \leq C r^{2}
$$

provided that $h \leq r$. Furthermore

$$
\begin{aligned}
\int_{\Omega} E[\tilde{H}(X(t ; \alpha))]^{2} d \alpha & \leq \int_{\Omega} \int_{B(x, 2 r)} G(y, t ; \alpha, 0) d y d \alpha \\
& \leq \int_{B(x, 2 r)} d y=4 \pi r^{2} .
\end{aligned}
$$

Therefore $\sum_{i} \operatorname{Var} Y_{i} \leq C r^{2} h^{2}$ if $r \geq h$.

According to Bennett's inequality, it follows that

$$
\begin{aligned}
P\{\mid & \left.\sum_{i} Y_{i}|\geq C r h| \ln h \mid\right\} \\
& \leq 4 \exp \left\{-\frac{1}{4}(C r h|\ln h|)^{2} \cdot C_{1} r^{-2} h^{-2} \cdot B\left[h^{2} C r h|\ln h| C_{1} r^{-2} h^{-2}\right]\right\} \\
& \leq \exp \left\{-C_{2} C^{2}|\ln h|^{2} \cdot B\left[C_{2} C r^{-1} h|\ln h|\right]\right\} \\
& \leq \exp \left\{-C_{3} C|\ln h|^{2}\right\} \\
& =h^{C_{3} C|\ln h|}
\end{aligned}
$$

provided that $r^{-1} h|\ln h|$ stays bounded.

Hence the fluctuation of $h^{2} \cdot \mathscr{N}(x, r, t)$ is

$$
|(I I)| \preceq C r h|\ln h| \leq C r^{2}
$$

if $h|\ln h| \leq r$. This completes the proof of the lemma.

We will apply the above lemma with $r \sim \delta$. The condition $r \geq h|\ln h|$ is satisfied if we choose $\delta=h^{q}, 0 \leq q \leq 1$. Next, we give a generalization of (5.11) on p. 47 of B-M.

Lemma 9. Let $M_{i j}^{(l)}=\max _{|y| \leq C_{0} \delta} \max _{\mid \beta=l}\left|\partial^{\beta} K_{\delta}\left(X_{i}-X_{j}+y\right)\right|$. Then

$$
\sum_{j} M_{i j}^{(l)} h^{2} \preceq \begin{cases}C|\ln \delta| & \text { if } l=1 \\ C \delta^{-1} & \text { if } l=2 .\end{cases}
$$


Proof. We prove the case $l=1$ and $C_{0}=1$ in detail. The proofs for general $C_{0}$ and $l=2$ are almost the same. We write

$$
\begin{gathered}
\begin{aligned}
\sum_{j} M_{i j}^{(1)} h^{2} & =\sum_{\left|X_{j}-X_{i}\right| \leq 2 \delta} M_{i j}^{(1)} h^{2}+\sum_{2 \delta<\left|X_{j}-X_{i}\right| \leq 2} M_{i j}^{(1)} h^{2}+\sum_{\left|X_{j}-X_{i}\right|>2} M_{i j}^{(1)} h^{2} \\
& =(I)+(I I)+(I I I) .
\end{aligned} \\
(I) \leq \bar{C} \cdot(2 \delta)^{2} \cdot C_{1} \delta^{-2} \leq C, \text { by the assumption and (i) of Lemma } 4 .
\end{gathered}
$$

In order to estimate $(I I)$ and $(I I I)$, we notice that $\left|X_{j}-X_{i}\right| \geq 2 \delta$ implies that

$$
\left|X_{j}-X_{i}+y\right| \geq\left|X_{j}-X_{i}\right|-|y| \geq \frac{1}{2}\left|X_{j}-X_{i}\right|
$$

It follows that

$$
(I I I) \leq C_{1} \sum_{\left|X_{j}-X_{i}\right| \geq 2} 4\left|X_{j}-X_{i}\right|^{-2} h^{2} \leq C_{1} \sum_{\left|X_{j}-X_{i}\right|>2} h^{2} \leq C
$$

and

$$
(I I) \leq C \sum_{2 \delta<\left|X_{j}-X_{i}\right| \leq 2}\left|X_{j}-X_{i}\right|^{-2} h^{2} .
$$

To estimate (28) we decompose the ring $\mathscr{R}=\left\{x: 2 \delta<\left|x-X_{i}\right| \leq 2\right\}$ into $N-2$ concentric rings $\mathscr{R}_{n}=\left\{x:(n+1) \delta<\left|x-X_{i}\right| \leq(n+2) \delta\right\}, 1 \leq n \leq N-2$ where $N=[2 / \delta]$ is the least integer greater than or equal to $2 / \delta$. Let $a_{n}=$ $\mathcal{N}\left(X_{i},(n+1) \delta, t\right)$ be the number of the vortices in the ball $B\left(X_{i},(n+1) \delta\right)$.

$$
\begin{aligned}
(I I) & \leq \sum_{X_{j} \in \mathscr{h}} C_{1} \cdot 4\left|X_{j}-X_{i}\right|^{-2} h^{2} \\
& \leq C_{2} \sum_{n=1}^{N-2} \sum_{X_{j} \in \mathscr{R}_{n}}\left|X_{j}-X_{i}\right|^{-2} h^{2} \\
& \leq C_{3} \sum_{n=2}^{N-1}(n \delta)^{-2}\left(a_{n}-a_{n-1}\right) h^{2} \\
& =C_{3}\left\{h^{2}\left[\frac{a_{N-1}}{N^{2} \delta^{2}}-\frac{a_{1}}{\delta^{2}}\right]-\sum_{n=1}^{N-1}\left[\frac{1}{(n+1)^{2} \delta^{2}}-\frac{1}{n^{2} \delta^{2}}\right] a_{n} h^{2}\right\} \\
& \leq C_{3}\left\{C_{4}+\sum_{n=1}^{N-1}\left[\frac{1}{\delta^{2}} \cdot \frac{(2 n+1)}{n^{2}(n+1)^{2}} \cdot C_{5}(n+1)^{2} \delta^{2}\right]\right\} \\
& \leq C_{3}\left\{C_{4}+C_{6} \sum_{n=1}^{N-1} \frac{1}{n}\right\} \\
& \leq C_{7}(1+\ln N) \\
& \leq C|\ln \delta|
\end{aligned}
$$

where (29) is obtained through summation by parts and (30) follows from Lemma 8. This completes the proof of the lemma. 
Finally we are ready to estimate $v^{(1)}$. We write

$$
\begin{aligned}
v_{i}^{(1)} & =\sum_{j}\left[K_{\delta}\left(X_{i}-\tilde{X}_{j}\right)-K_{\delta}\left(X_{i}-X_{j}\right)\right] \omega_{j} h^{2} \\
& =\sum_{j} \nabla K_{\delta}\left(X_{i}-X_{j}+Y_{i j}\right) \cdot e_{j} \omega_{j} h^{2}
\end{aligned}
$$

where $e_{j}=X_{j}-\tilde{X}_{j}$ and we ignore the fact that $Y_{i j}$ may depend on the components. Let $Z_{i} \in \delta \cdot \mathbf{Z}^{2}$ be the closest lattice point to $X_{i}$. If there is more than one lattice point closest to $X_{i}$, then we chose one of them arbitrarily. We write

$$
v_{i}^{(1)}=\sum_{j} \nabla K_{\delta}\left(Z_{i}-Z_{j}\right) \cdot e_{j} \omega_{j} h^{2}+r_{i}^{(1)}
$$

where

$$
r_{i}^{(1)}=\sum_{j}\left[\nabla K_{\delta}\left(X_{i}-X_{j}+Y_{i j}\right)-\nabla K_{\delta}\left(Z_{i}-Z_{j}\right)\right] \cdot e_{j} \omega_{j} h^{2}
$$

For each $z_{k}=\delta \cdot k=\delta \cdot\left(k_{1}, k_{2}\right)$, we define $f_{k}$ to be the average of all $e_{j} \omega_{j}$ where $X_{j}$ is in the square $Q_{k}$. Namely,

$$
f_{k}=\delta^{-2} \sum_{X_{j} \in Q_{k}} e_{j} \omega_{j} h^{2}
$$

with the convention that $f_{k}=0$ if $Q_{k}$ contains none of the vortices $X_{j}$. It follows from Lemma 8 that

$$
\begin{aligned}
\left\|f_{k}\right\|_{L_{i}^{p}}^{p} & =\sum_{k} \delta^{-2 p+2}\left|\sum_{X_{j} \in Q_{k}} e_{j} \omega_{j} h^{2}\right|^{p} \\
& \preceq \sum_{k} \delta^{-2 p+2} \cdot\left(C \delta^{2}\right)^{p-1} \sum_{X_{j} \in Q_{k}}\left|e_{j} \omega_{j}\right|^{p} h^{2} \\
& =C^{p-1}\left\|e_{j} \omega_{j}\right\|_{L_{h}^{p}}^{p} .
\end{aligned}
$$

Furthermore,

$$
\left\|\sum_{j} \nabla K_{\delta}\left(Z_{i}-Z_{j}\right) \cdot e_{j} \omega_{j} h^{2}\right\|_{L_{h}^{p}} \preceq C\left\|\sum_{k^{\prime}} \nabla K_{\delta}\left(z_{k}-z_{k^{\prime}}\right) \cdot f_{k^{\prime}} \delta^{2}\right\|_{L_{\delta}^{\prime \prime}}
$$


since

$$
\begin{aligned}
& \left\|\sum_{j} \nabla K_{\delta}\left(Z_{i}-Z_{j}\right) \cdot e_{j} \omega_{j} h^{2}\right\|_{L_{h}^{p}}^{p}=\left\|\sum_{k^{\prime} \in \mathbf{Z}^{2}} \nabla K_{\delta}\left(Z_{i}-z_{k^{\prime}}\right) \cdot f_{k^{\prime}} \delta^{2}\right\|_{L_{h}^{p}}^{p} \\
& \quad=\sum_{i}\left|\sum_{k^{\prime}} \nabla K_{\delta}\left(Z_{i}-z_{k^{\prime}}\right) \cdot f_{k^{\prime}} \delta^{2}\right|^{p} \cdot h^{2} \\
& \quad=\sum_{k} \#\left(X_{i} \in Q_{k}\right) \cdot\left|\sum_{k^{\prime}} \nabla K_{\delta}\left(z_{k}-z_{k^{\prime}}\right) \cdot f_{k^{\prime}} \delta^{2}\right|^{p} \cdot h^{2} \\
& \quad \preceq C \sum_{k}\left|\sum_{k^{\prime}} \nabla K_{\delta}\left(z_{k}-z_{k^{\prime}}\right) \cdot f_{k^{\prime}} \delta^{2}\right|^{p} \cdot \delta^{2} \\
& \quad=C \|\left.\sum_{k^{\prime}} \nabla K_{\delta}\left(z_{k}-z_{k^{\prime}}\right) \cdot f_{k^{\prime}} \delta^{2}\right|_{L_{\delta}^{p}} ^{p}
\end{aligned}
$$

by Lemma 8 .

We can now follow the same procedure in B-M which is outlined above to rewrite the sum $\sum_{k} \nabla K_{\delta}\left(z_{i}-z_{k}\right) f_{k} \delta^{2}$ by the integral $\int \widetilde{\mathscr{K}}\left(x, x^{\prime}\right) \tilde{f}\left(x^{\prime}\right) d x^{\prime}$ where $\tilde{f}=f_{k}$ on the square $Q_{k}$ and $\widetilde{\mathscr{K}}\left(x, x^{\prime}\right)=\nabla K_{\delta}\left(z_{i}-z_{k}\right), x \in Q_{i}$, $x^{\prime} \in Q_{k}$. It follows from the argument in B-M (pp. 47-48) that

$$
\left\|\sum_{k \in \mathbf{Z}^{2}} \nabla K_{\delta}\left(z_{i}-z_{k}\right) \cdot f_{k} \delta^{2}\right\|_{L_{\delta}^{p}} \leq C\left\|f_{k}\right\|_{L_{\delta}^{p}}
$$

which implies that

$$
\left\|\sum_{j} \nabla K_{\delta}\left(Z_{i}-Z_{j}\right) \cdot e_{j} \omega_{j} h^{2}\right\|_{L_{h}^{p}} \preceq C\left\|e_{j} \omega_{j}\right\|_{L_{h}^{p}}
$$

provided that $\delta \geq h|\ln h|$. By the Mean Value Theorem

$$
r_{i}^{(1)}=\sum_{j}\left[\nabla^{2} K_{\delta}\left(X_{i}-X_{j}+Y_{i j}+Y_{i j}^{\prime \prime}\right) \cdot Y_{i j}^{\prime}\right] \cdot e_{j} \omega_{j} h^{2}
$$

where $Y_{i j}^{\prime}=Y_{i j}+\left(X_{i}-Z_{i}\right)-\left(X_{j}-Z_{j}\right)$ and we ignore that $Y_{i j}^{\prime}$ and $Y_{i j}^{\prime \prime}$ may depend on the components. Since $\left|Y_{i j}\right| \leq \delta,\left|Y_{i j}^{\prime \prime}\right| \leq\left|Y_{i j}^{\prime}\right| \leq 4 \delta$,

$$
\left|r_{i}^{(1)}\right| \leq \sum_{j} M_{i j}^{(2)} \cdot 4 \delta \cdot\left|e_{j} \omega_{j}\right| h^{2} .
$$

By Young's inequality (see Folland $[7, \S 0 . C]$ ) and Lemma 9 with $C_{0}=5$, we conclude that

$$
\begin{aligned}
\left\|r_{i}^{(1)}\right\|_{L_{h}^{p}} & \leq 4 \delta \cdot \max \left\{\sum_{j} M_{i j}^{(2)} h^{2}, \sum_{i} M_{i j}^{(2)} h^{2}\right\} \cdot\left\|e_{j} \omega_{j}\right\|_{L_{h}^{p}} \\
& \preceq C\left\|e_{j} \omega_{j}\right\|_{L_{h}^{p}}
\end{aligned}
$$

This finishes the estimation for $v^{(1)}$. 
To estimate $v^{(2)}$, we apply the Mean Value Theorem

$$
\begin{aligned}
v_{i}^{(2)} & =\sum_{j} \nabla K_{\delta}\left(X_{i}-X_{j}+Y_{i j}\right) \cdot\left(\tilde{X}_{i}-X_{i}\right) \cdot \omega_{j} h^{2} \\
& =\left[\sum_{j} \nabla K_{\delta}\left(X_{i}-X_{j}+Y_{i j}\right) \cdot \omega_{j} h^{2}\right] \cdot\left(\tilde{X}_{i}-X_{i}\right) .
\end{aligned}
$$

We want to show that

$$
\max _{i}\left|\sum_{j} \nabla K_{\delta}\left(X_{i}-X_{j}+Y_{i j}\right) \cdot \omega_{j} h^{2}\right| \preceq C .
$$

Since $\left|\nabla K_{\delta}\left(X_{i}-X_{j}+Y_{i j}\right)-\right| \nabla K_{\delta}\left(X_{i}-X_{j}\right) \mid \leq \delta M_{i j}^{(2)}$, it is enough to prove the uniform boundedness of $\sum_{j} \nabla K_{\delta}\left(X_{i}-X_{j}\right) \cdot \omega_{j} h^{2}$ by Lemma 9 and Young's inequality as in the estimation for $r^{(1)}$. The proof is essentially the same as the consistency estimate except for $K_{\delta}$ being replaced by $\nabla K_{\delta}$. For any $x \in \mathbf{R}^{2}$ we may write

$$
\begin{aligned}
\sum_{j} \nabla K_{\delta}\left(x-X_{j}\right) \cdot \omega_{j} h^{2}= & \sum_{j} E \nabla K_{\delta}\left(x-X_{j}\right) \cdot \omega_{j} h^{2} \\
& +\sum_{j}\left[\nabla K_{\delta}\left(x-X_{j}\right)-E \nabla K_{\delta}\left(x-X_{j}\right)\right] \cdot \omega_{j} h^{2} \\
= & (I I I)+(I V) .
\end{aligned}
$$

By applying Lemma 1 with $f(y)=\nabla K_{\delta}(x-y), g(\alpha)=\omega(\alpha, 0)$, we can approximate $(I I I)$ by the integral

$$
\int_{\mathbf{R}^{2}} E\left[\nabla K_{\delta}(x-X(t ; \alpha))\right] \cdot \omega(\alpha, 0) d \alpha=\int_{\mathbf{R}^{2}} \nabla K_{\delta}(x-y) \cdot \omega(y, t) d y
$$

with an error $C(h / \delta)^{L}$ which follows from Lemmas 4 and 5. (III) is uniformly bounded since

$$
\begin{aligned}
\left|\int_{\mathbf{R}^{2}} \nabla K_{\delta}(x-y) \cdot \omega(y, t) d y\right| & =\left|\int_{\mathbf{R}^{2}} K_{\delta}(x-y) \cdot \nabla \omega(y, t) d y\right| \\
& \leq\|\nabla \omega\|_{L^{\infty}}\left\|K_{\delta} \mid\right\|_{L^{1}(B)}+\|\nabla \omega\|_{L^{1}}\left\|K_{\delta}\right\|_{L^{\infty}\left(\mathbf{R}^{2} \backslash B\right)}
\end{aligned}
$$

is uniformly bounded with respect to $x$ where $B=\left\{x \in \mathbf{R}^{2}:|x|<1\right\}$.

We begin to estimate $(I V)$. Let

$$
Y_{j}=\nabla K_{\delta}\left(x-X_{j}\right) \cdot \omega_{j} h^{2}-E \nabla K_{\delta}\left(x-X_{j}\right) \cdot \omega_{j} h^{2} .
$$

We assume $h|\ln h| \leq \delta$ as before. It follows that

$$
\left|Y_{j}\right| \leq C h^{2} \delta^{-2} \leq C|\ln h|^{-2} \stackrel{\text { def }}{=} M .
$$

To bound the variance

$$
V=\sum_{j} \operatorname{Var} Y_{j} \leq h^{2} \sum_{j} E\left|\nabla K_{\delta}\left(x-X_{j}\right)\right|^{2} \cdot \omega_{j}^{2} h^{2},
$$


we once again apply Lemma 1 to approximate the sum in (31) by the integral

$$
\int_{\mathbf{R}^{2}}\left|\nabla K_{\delta}(x-y)\right|^{2}\left[\int_{\Omega} G(y, t ; \alpha, 0) \omega^{2}(\alpha, 0) d \alpha\right] d y
$$

within an error $C h^{L} \delta^{-2-L} \leq C \delta^{-2}$. Therefore $V \leq C h^{2} \delta^{-2} \leq C|\ln h|^{-2}$. From Bennett's inequality we obtain

$$
\begin{aligned}
P\left\{\left|\sum_{j} Y_{j}\right| \geq C\right\} & \leq 4 \exp \left\{-\frac{1}{4} C^{2} V^{-1} B\left(M C V^{-1}\right)\right\} \\
& \leq \exp \left\{-C^{2} C_{1}|\ln h|^{2} B\left(C_{2} C\right)\right\} \\
& \leq \exp \left\{-C_{3} C^{2}|\ln h|^{2} \cdot C^{-1}\right\} \\
& \leq \exp \left\{-C_{3} C|\ln h|^{2}\right\} \\
& =h^{C_{3} C|\ln h|} .
\end{aligned}
$$

Hence for any fixed time $t, \sum_{j} \nabla K_{\delta}\left(X_{i}-X_{j}\right) \cdot \omega_{j} h^{2}$ is uniformly bounded by $C$ except for an event of probability less than $h^{C_{4} C|\ln h|}$.

We need to extend the previous stability result to all time $t \in\left[0, T_{*}\right]$. Here is our strategy. Let $t_{n}, n=0, \ldots, N$, divide $\left[0, T_{*}\right]$ into $N$ subintervals with lengths less than $h^{l}$ for some $l>0$ to be determined later. Since the stability estimate holds for any fixed time except for an event of probability less than $h^{C_{1} C|\ln h|}$, it holds on $\left\{t_{n}\right\}_{n=1}^{N}$ except for an event of probability less than $C_{2} h^{C_{1} C|\ln h|-l}$ where $C_{1} C>l$ by choosing $C$ large enough. The length of each time interval approaches zero as the number of vortices approaches infinity. Therefore, if we can prove that the positions of the vortices $\widetilde{X}_{i}(t), X_{i}(t)$ for $t \in\left[t_{n}, t_{n+1}\right]$ are close to their own positions at time $t=t_{n}$, then we can pass the stability estimate on $t_{0}, \ldots, t_{N}$ to the stability estimate for all time. To be more precise, we write

$$
\begin{aligned}
\tilde{X}_{i}(t)-X_{i}(t) & =\left\{\tilde{X}_{i}\left(t_{n}\right)+\left[\tilde{X}_{i}(t)-\tilde{X}_{i}\left(t_{n}\right)+X_{i}\left(t_{n}\right)-X_{i}(t)\right]\right\}-X_{i}\left(t_{n}\right) \\
& =\left\{\tilde{X}_{i}\left(t_{n}\right)+Y_{i}(t)\right\}-X_{i}\left(t_{n}\right) \\
& =\tilde{\tilde{X}}_{i}(t)-X_{i}\left(t_{n}\right)
\end{aligned}
$$

for $t \in\left[t_{n}, t_{n+1}\right]$. Since our stability estimate (with Lemmas 8 and 9) requires only statistics of $X_{i}\left(t_{n}\right)$ and treat $\widetilde{X}_{i}\left(t_{n}\right)$ as small perturbation from $X_{i}\left(t_{n}\right)$, it makes no difference to add another small perturbation $Y_{i}(t)$ to $\tilde{X}_{i}\left(t_{n}\right)$ and the stability estimate

$$
\left\|\tilde{u}_{i}^{h}(t)-u_{i}^{h}(t)\right\|_{L_{h}^{p}} \preceq C\left\|\widetilde{\widetilde{X}}_{i}(t)-X_{i}\left(t_{n}\right)\right\|_{L_{h}^{p}}=C\left\|\tilde{X}_{i}(t)-X_{i}(t)\right\|_{L_{h}^{p}}
$$

holds for all $t, t_{n}<t<t_{n+1}$, as long as $\left|Y_{i}(t)\right| \preceq$ constant $\cdot \delta$ since our estimate does not make use of the statistics of $\widetilde{X}_{i}(t)$.

To prove $\max _{n} \max _{t_{n} \leq t \leq t_{n+1}}\left|X_{i}(t)-X_{i}\left(t_{n}\right)\right| \preceq \delta$, we need the following elementary property of Wiener processes. 
Lemma 10. Let $W(t)$ be a standard Wiener process in $\mathbf{R}^{d}$. Then

$$
P\left\{\max _{t \leq s \leq t+\Delta t}|W(s)-W(t)| \geq b\right\} \leq C_{1}(\sqrt{\Delta t} / b) \exp \left(-C_{2} b^{2} / \Delta t\right)
$$

where $b>0$ and the positive constants $C_{1}, C_{2}$ depend only on $d$.

See Freedman [7, p. 18] for a proof in $\mathbf{R}$. Since

$$
X_{i}(t)-X_{i}(s)=\int_{s}^{t} u\left(X_{i}(\tau), \tau\right) d \tau+\sqrt{2 \nu}\{W(t)-W(s)\},
$$

it follows that for all $t \in\left[t_{n}, t_{n+1}\right]$

$$
\begin{aligned}
\left|X_{i}(t)-X_{i}\left(t_{n}\right)\right| & \leq C_{1}\left|t-t_{n}\right|+\sqrt{2 \nu}\left|W(t)-W\left(t_{n}\right)\right| \\
& \leq C h^{l}+\sqrt{2 \nu}\left|W(t)-W\left(t_{n}\right)\right| .
\end{aligned}
$$

By Lemma 10

$$
P\left\{\max _{t_{n} \leq t \leq t_{n+1}}\left|W(t)-W\left(t_{n}\right)\right| \geq h\right\} \leq C_{1} h^{(l / 2)-1} \exp \left[-C_{2} h^{-l+2}\right]
$$

which implies that for $l>2$,

$$
P\left\{\max _{n} \max _{t_{n} \leq t \leq t_{n+1}}\left|W(t)-W\left(t_{n}\right)\right| \geq h\right\} \leq C_{1} h^{-(l+2) / 2} \exp \left\{-C_{2} h^{-l+2}\right\} \rightarrow 0
$$

faster than any polynomial in $h$ as $h \rightarrow 0$. This justifies that

$$
\begin{aligned}
\max _{n} \max _{t_{n} \leq t \leq t_{n+1}}\left|X_{i}(t)-X_{i}\left(t_{n}\right)\right| & \leq C h^{l}+\sqrt{2 \nu}\left|W(t)-W\left(t_{n}\right)\right| \\
& \preceq C h^{l}+\nu^{1 / 2} h \leq \delta
\end{aligned}
$$

and the proof of the stability lemma for all time is completed.

\section{CONVERGENCE}

We first prove the convergence of the particle paths and the discrete velocity. In order to do that we need to extend the consistency estimate for the discete velocity to all time. It can be derived simply by combining the consistency estimate for a finite number of times $0=t_{0}<t_{1}<\cdots<t_{N}=T$ and the stability estimate for all time where $\max _{n}\left|t_{n+1}-t_{n}\right|<h^{l}$ with $l>2$. For each $t \in\left[t_{n}, t_{n+1}\right)$, we apply the triangle inequality to obtain

$$
\begin{aligned}
\left\|u_{i}^{h}(t)-u\left(X_{i}(t), t\right)\right\|_{L_{h}^{p}} \leq & \left\|u_{i}^{h}(t)-u_{i}^{h}\left(t_{n}\right)\right\|_{L_{h}^{p}}+\left\|u_{i}^{h}\left(t_{n}\right)-u\left(X_{i}\left(t_{n}\right), t_{n}\right)\right\|_{L_{h}^{p}} \\
& +\left\|u\left(X_{i}\left(t_{n}\right), t_{n}\right)-u\left(X_{i}(t), t\right)\right\|_{L_{h}^{p}} \\
\leq & C_{1}\left\|X_{i}(t)-X_{i}\left(t_{n}\right)\right\|_{L_{h}^{p}}+C_{2}\left[\delta^{m}+(h / \delta)^{L} \delta+h|\ln h|\right] \\
\preceq & C\left[\delta^{m}+(h / \delta)^{L} \delta+h|\ln h|\right]
\end{aligned}
$$

by the estimate $\left|X_{i}(t)-X_{i}\left(t_{n}\right)\right| \preceq C\left[h^{l}+\nu^{1 / 2} h\right]$ and the assumption that $u$ has bounded derivatives. Therefore

$$
\max _{0 \leq t \leq T}\left\|u_{i}^{h}(t)-u\left(X_{i}(t), t\right)\right\|_{L_{h}^{p}} \preceq C\left[\delta^{m}+(h / \delta)^{L} \delta+h|\ln h|\right] .
$$


Similarly,

$$
\max _{0 \leq t \leq T}\left\|u^{h}\left(z_{k}, t\right)-u\left(z_{k}, t\right)\right\|_{L_{h}^{p}} \preceq C\left[\delta^{m}+(h / \delta)^{L} \delta+h|\ln h|\right]
$$

for the lattice points $z_{k}=h \cdot k$ in any ball $B\left(R_{0}\right)$. This finishes the (discrete) consistency estimate for all time. Notice that the $L^{\infty}$ estimate is excluded since we applied the stability lemma.

The convergence of particle paths and the discrete velocity can be proved by the same argument as in B-M. Let $e_{i}(t)=\widetilde{X}_{i}(t)-X_{i}(t)$. We have

$$
d e_{i}(t)=\left[\tilde{u}_{i}^{h}(t)-u\left(X_{i}(t), t\right)\right] d t
$$

and the differential inequality

$$
\left\|\frac{d e_{i}}{d t}\right\|_{L_{h}^{p}} \preceq C\left[\left\|e_{i}(t)\right\|_{L_{h}^{p}}+\delta^{m}+(h / \delta)^{L} \delta+h|\ln h|\right]
$$

by the stability lemma and the consistency estimate (32). Since $e_{i}(0)=0$, it follows from (34) that $\left\|e_{i}(t)\right\|_{L_{h}^{p}} \preceq y(t), 0 \leq t \leq T_{*}$, where $y$ is the solution of the ODE

$$
\frac{d y}{d t}=C\left[y+\delta^{m}+(h / \delta)^{L} \delta+h|\ln h|\right], \quad y(0)=0 .
$$

Therefore $\left\|e_{i}(t)\right\|_{L_{h}^{p}} \preceq \bar{C}\left[\delta^{m}+(h / \delta)^{L} \delta+h|\ln h|\right], 0 \leq t \leq T_{*}$.

To complete the proof, we need to justify that $\left|e_{i}(t)\right| \preceq \delta$ for all $i$ and $0 \leq t \leq T$. Since $h^{2} \cdot \max _{i}\left|e_{i}\right|^{p} \leq\left(\left\|e_{i}\right\|_{L_{h}^{p}}\right)^{p}$, we have

$$
\max _{i}\left|e_{i}\right| \leq h^{-2 / p}\left\|e_{i}\right\|_{L_{h}^{p}} \preceq C h^{1-(2 / p)}|\ln h| \leq \frac{\delta}{2}
$$

for $t<T_{*}$ by choosing $p$ large enough, $\delta=h^{q}$ with appropriate $q<1$, and $h$ small enough. Therefore, $\max _{i}\left|e_{i}\right|$ can hardly reach $\delta$ and all the estimates hold for $T_{*}=T$.

For the convergence of discrete velocity, we have

$$
\begin{aligned}
\left\|\tilde{u}_{i}^{h}(t)-u\left(X_{i}(t), t\right)\right\|_{L_{h}^{p}} & \leq\left\|\tilde{u}_{i}^{h}(t)-u_{i}^{h}(t)\right\|_{L_{h}^{p}}+\left\|u_{i}^{h}(t)-u\left(X_{i}(t), t\right)\right\|_{L_{h}^{p}} \\
& \preceq C_{1}\left\|\tilde{X}_{i}(t)-X_{i}(t)\right\|_{L_{h}^{p}}+C_{2}\left[\delta^{m}+(h / \delta)^{L} \delta+h|\ln h|\right] \\
& \preceq C\left[\delta^{m}+(h / \delta)^{L} \delta+h|\ln h|\right]
\end{aligned}
$$

by applying the consistency estimate (32), the stability lemma, and the convergence of particle paths.

For the convergence of the continuous velocity, we consider the lattice points $B^{h}=\left(h \cdot \mathbf{Z}^{2}\right) \cap B\left(R_{0}\right)$ and apply the triangle inequality to obtain

$$
\begin{aligned}
\left|\tilde{u}^{h}(x, t)-u(x, t)\right| \leq & \left|\tilde{u}^{h}(x, t)-u^{h}(x, t)\right|+\left|u^{h}(x, t)-u(x, t)\right| \\
\leq \leq & \left|\tilde{u}^{h}(x, t)-\tilde{u}^{h}\left(z_{k}, t\right)\right|+\left|\tilde{u}^{h}\left(z_{k}, t\right)-u^{h}\left(z_{k}, t\right)\right| \\
& +\left|u^{h}\left(z_{k}, t\right)-u\left(z_{k}, t\right)\right|+\left|u\left(z_{k}, t\right)-u(x, t)\right| \\
= & (I)+(I I)+(I I I)+(I V)
\end{aligned}
$$


where $z_{k} \in B^{h}$ is the lattice point closest to $x \in B\left(R_{0}\right)$. The set of all points $x$ closest to $z_{k}$ is the square $Q_{k}$ centered at $z_{k}$ with its sides parallel to the axes and of length $h$. The continuous velocities $\tilde{u}^{h}(\cdot, t)$ and $u(\cdot, t)$ are approximated by the piecewise constant functions which take values $\tilde{u}^{h}\left(z_{k}, t\right)$ and $u\left(z_{k}, t\right)$ on $Q_{k}$ respectively. The terms $(I)$ and $(I V)$ are the errors introduced by these approximations. (III) is handled by the consistency estimate (33) and $|(I V)| \leq C h$ follows from the boundedness of the derivatives of $u$. To estimate $(I)$ we apply the Mean Value Theorem to write

$$
\begin{aligned}
\tilde{u}^{h}(x, t)-\tilde{u}^{h}\left(z_{k}, t\right) & =\sum_{i} K_{\delta}\left(x-\tilde{X}_{i}(t)\right) \omega_{i} h^{2}-\sum_{i} K_{\delta}\left(z_{k}-\tilde{X}_{i}(t)\right) \omega_{i} h^{2} \\
& =\sum_{i} \nabla K_{\delta}\left(z_{k}-\tilde{X}_{i}(t)+Y_{k i}\right) \cdot\left(x-z_{k}\right) \omega_{i} h^{2} \\
& =\left[\sum_{i} \nabla K_{\delta}\left(z_{k}-X_{i}(t)+Z_{k i}\right) \cdot \omega_{i} h^{2}\right] \cdot\left(x-z_{k}\right)
\end{aligned}
$$

where $Z_{k i}=X_{i}(t)-\tilde{X}_{i}(t)+Y_{k i}$ and we ignore that $Y_{k i}$ may depend on the components. By the convergence of the particle paths, we have

$$
\left|Z_{k i}\right| \leq\left|X_{i}(t)-\tilde{X}_{i}(t)\right|+\left|Y_{k i}\right| \preceq \delta+\left|x-z_{k}\right| \leq 2 \delta .
$$

We use the same arguments as in the estimate for $v^{(2)}$ in the stability lemma to obtain that

$$
\max _{k}\left|\sum_{i} \nabla K_{\delta}\left(z_{k}-X_{i}(t)+Z_{k i}\right) \cdot \omega_{i} h^{2}\right| \preceq C .
$$

Finally,

$$
\begin{aligned}
(I I) & =\left|\sum_{i}\left[K_{\delta}\left(z_{k}-\tilde{X}_{i}(t)\right)-K_{\delta}\left(z_{k}-X_{i}(t)\right)\right] \omega_{i} h^{2}\right| \\
& =\left|\sum_{i} \nabla K_{\delta}\left(z_{k}-X_{i}(t)+Y_{k i}\right) \cdot e_{i} \omega_{i} h^{2}\right|
\end{aligned}
$$

and it can be treated in the same way as that of $v^{(1)}$ in the stability lemma. The extension to all time is obvious, too. This completes the proof for the convergence of the continuous velocity.

\section{ACKNOWLEDGMENT}

This paper is based on a part of the author's Ph.D. thesis at the University of California at Berkeley. The author wishes to thank his thesis advisor, Professor Andrew Majda, for his invaluable feedback, support, and suggestions on this work. The author also wishes to thank Professors Alexander Chorin and Ole Hald for their support and encouragement. 


\title{
REFERENCES
}

1. C. Anderson and C. Greengard, On vortex methods, SIAM J. Numer. Anal. 22 (3) (1985), pp. 413-440.

2. J. T. Beale and A. Majda, Rates of convergence for viscous splitting of the Navier-Stokes equations, Math. of Comp. 37 (1981), pp. 243-260.

3. $\frac{1}{1-27}$, Vortex methods. I: Convergence in three dimensions, Math. of Comp. 39 (1982), pp.

4. __ Vortex methods. II: Higher order accuracy in two and three dimensions, Math. of Comp. 39 (1982), pp. 29-52.

5. A. J. Chorin, Numerical study of slightly viscous flow, J. Fluid Mech. 57 (1973), pp. 785-796.

6. G. H. Cottet, Méthodes particulaires pour l'équation d'Euler dans le plan, Thèse de $3 \mathrm{e}$ cycle, l'Université Pièrre et Marie Curie, Paris, France, 1982.

7. G. Folland, Introduction to partial differential equations, Princeton Univ. Press, Princeton, N. J., 1978.

8. D. A. Freedman, Brownian motion and diffusion, Holden-Day, San Francisco, 1971.

9. A. Friedman, Stochastic differential equations and applications, Academic Press, New York, 1975.

10. J. Goodman, Convergence of the random vortex method, Comm. Pure Appl. Math. 40 (2) (1987), pp. 189-220.

11. O. Hald, The convergence of vortex method, SIAM J. Numer. Anal. 16 (1979), pp. 726-755.

12. D.-G. Long, Convergence of a random vortex method in three dimensions, preprint, 1988.

13. _ _ Time discretization of the random vortex methods (to appear).

14. _ Viscosity dependence of the sampling errors in the random vortex methods (to appear).

15. C. Marchioro and M. Pulvirenti, Hydrodynamics in two dimensions and vortex theory, Comm. Math. Phys. 84 (1982), pp. 483-503.

16. D. Pollard, Convergence of stochastic processes, Springer-Verlag, New York, 1984.

\begin{abstract}
A theoretical framework for analyzing the random vortex method is presented. It extends and modifies the analysis of the inviscid vortex method in a natural and unified manner.

The rate of convergence of the random vortex method in two dimensions is obtained by analyzing the consistency error and justifying the stability estimate. The sampling error introduced by the random motions of finitely many vortices is the dominant component of the consistency error in terms of order. It is estimated by applying Bennett's inequality.
\end{abstract}

Mathematical Sciences Institute, Cornell University, Ithaca, New York 14850 